\title{
PREDICTING THE PROPERTIES OF SECOND CYCLE HYBRIDS PRODUCED BY INTERCROSSING RANDOM SAMPLES OF RECOMBINANT INBRED LINES
}

\author{
J. F. F. DE TOLEDO, H. S. POONI AND J. L. JINKS \\ Department of Genetics, University of Birmingham, P.O. Box 363, Birmingham B15 $2 T T$

\section{SUMMARY}

Distributive properties of the second cycle hybrids that are produced by intercrossing the recombinant inbreds extractable from the $F_{2}$ of a cross between two pure breeding lines can be predicted from the early generations of the original cross. Hence the frequency of such hybrids that will outperform the extreme recombinant inbreds or the original $F_{1}$ can be predicted. Basic generations and triple test cross families provide the most reliable estimates of the predictors and therefore should be used whenever possible although, in the presence of linkage, randomly mated $F_{2}$ 's may give improved predictions. Simpler experiments consisting of the parental varieties and their $F_{1}$ and $F_{2}$ generations, however, provide all the information that is likely to be necessary for most practical purposes.

The predictive power of the new approach is demonstrated on material extracted from the cross of varieties 1 and 5 of Nicotiana rustica. The predictors were estimated from the means and variances of $V_{1}, V_{5}, F_{1}$ and $F_{2}$ raised in six environments between 1973 and 1983 . The predicted frequencies of second cycle $F_{1}$ 's which outperform the extreme recombinant inbred lines derived from this cross are compared with those observed among 190 second cycle hybrids in a diallel between 20 recombinant inbreds derived from the same cross.

\section{INTRODUCTION}

The breeding value of a cross in respect of its production of superior recombinant inbred lines by pedigree inbreeding, single seed descent and dihaploidy can be readily and reliably predicted (Jinks and Pooni, 1976, 1980; Pooni and Jinks, 1978, 1981) using the basic generations and triple test cross families (Mather and Jinks, 1982; Kearsey and Jinks, 1968; Pooni and Jinks, 1979). The best of these recombinant inbreds may themselves be the appropriate end product of the breeding programme. However, second cycle $F_{1}$ hybrids obtained by intercrossing the recombinant inbreds may be superior end products and this may indicate the potential for further improvement of the inbreds by a second cycle of crossing and inbreeding. In this paper we shall, therefore, predict the properties of the second cycle hybrids produced by intercrossing the recombinant inbred lines derivable from the $F_{2}$ of a cross. We shall make these predictions using genetical information that has usually already been obtained for other purposes and illustrate the theory by analysing data recorded on the basic generations of the cross between varieties 1 and 5 of Nicotiana rustica. We shall use the predictions to assess the breeding value of a cross for producing second cycle $F_{1}$ hybrids which are superior to the best of the first cycle recombinant inbred lines. 


\section{Genotypes of InTEREst}

Diallel mating of all possible recombinant inbred lines which can be extracted from a cross between two pure breeding varieties by the method of pedigree inbreeding, single seed descent and dihaploidy produces a diallel table of $2^{K} \times 2^{K}$ genotypes. While the overall dimensions of this table depend upon $K$, the number of gene loci for which the inbreds differ for a particular trait, the $2^{K}$ genotypes entered on its leading diagonal are the recombinant inbreds which were used to produce this diallel. We have described the theory and practice of predicting the properties of these inbreds on numerous occasions (Jinks and Pooni, 1976, 1981; Pooni and Jinks, 1978, 1981; Pooni, Jinks and Cornish, 1977). Similarly, each of the $2^{K}$ genotypes on the off diagonal is heterozygous for all of the $K$-loci and therefore identical with the original $F_{1}$, whose properties are also known. The remaining $2^{(K-1)}\left(2^{K}-2\right)$ genotypes, which are diametrically duplicated in the upper and lower halves of the table, are the $F_{1}$ 's whose properties are hitherto unknown. These second cycle hybrids are of special interest because amongst them there may be unique genotypes which are capable of outperforming the best of the recombinant inbred lines or the original $\mathrm{F}_{1}$.

\section{Predictions for the Simple CASE}

To predict the properties of the second cycle hybrids we need to define their distribution in terms of genetical parameters which can be estimated from the early generations of a breeding programme. The joint distribution of the $2^{K-1}\left(2^{K}-2\right)$ recombinant hybrids described in section 2 and the $2^{K}$ original $F_{1}$ 's which cannot be separated from the former in practice, has the expected mean

$$
m+\frac{2^{K-1}}{2^{K}-1}[h]
$$

and variance

$$
\frac{\left(2^{K-1}-1\right)}{\left(2^{K}-1\right)} D+\frac{2^{K-1}\left(2^{K-1}-1\right)}{\left(2^{K}-1\right)^{2}} H-\frac{2^{K-1}}{\left(2^{K}-1\right)^{2}} \sum_{j<k}^{K} h_{j} h_{k}+E
$$

when an additive, dominance and additive environmental model is sufficient to explain the total variability (for definitions of the genetic parameters see Mather and Jinks, 1971, 1982). These expectations though dependent on " $K$ " in a complex manner approximate very closely with $m+\frac{1}{2}[h]$ and $\frac{1}{2} D+\frac{1}{4} H+E$, the mean and variance of the $\mathrm{F}_{2}$ generation of the original cross from which the recombinant inbreds were extracted, especially when $K$ is large $(K \geqq 6)$. Thus even when $K$ takes a small value (say $K=3$ ) the expectations of these statistics differ from $m+\frac{1}{2}[h]$ and $\frac{1}{2} D+\frac{1}{4} H+E$ by only $\frac{1}{14}[h]$ and $-\frac{1}{14} D-\frac{1}{196} H-\frac{4}{49} \sum_{j<k}^{K} h_{j} h_{k}$, respectively. Statistics $m+\frac{1}{2}[h]$ and $\frac{1}{2} D+\frac{1}{4} H+E$ will therefore provide estimates of the expected mean and variance of the recombinant hybrids that are satisfactory for all practical purposes.

Estimates of $m+\frac{1}{2}[h]$ and $\frac{1}{2} D+\frac{1}{4} H+E$ can be readily obtained from a number of breeding programmes. For example, the standard $P_{1}, P_{2}, F_{1}, F_{2}$, $B_{1}$ and $B_{2}$ families provide the most rapid and efficient estimates of both statistics (Mather and Vines, 1952) and a triple test cross in which the $F_{2}$ 
is backcrossed to $P_{1}, P_{2}$ and $F_{1}$ provides the most reliable estimates of $\frac{1}{2} D$ and $\frac{1}{4} H$ (Kearsey and Jinks, 1968; Pooni and Jinks, 1976). Since these are the commonest designs currently used by geneticists and.practical breeders, all the information required to predict the second cycle, recombinant $F_{1}$ hybrids will already be widely available. If, however, it is not, a small experiment including the original parental varieties and their $F_{1}$ and $F_{2}$ generations can be used to estimate the genetic and environmental components required for the predictions. From these estimates of the expected means and variances we can predict some useful properties of the recombinant hybrids. For example, we can obtain probabilities of hybrids which will score $>\overline{\mathrm{P}}_{1}$ or $<\overline{\mathrm{P}}_{2}$, the original parental varieties or outperform their $\overline{\mathrm{F}}_{1}$ if it shows heterosis. But more important, we can predict the probability of obtaining hybrids which outperform the extreme recombinant inbreds ( $>\overline{\mathrm{P}}_{\text {largest }}$ and $<\overline{\mathrm{P}}_{\text {smallest }}$ referred to as $>\overline{\mathrm{P}}_{l}$ and $<\overline{\mathrm{P}}_{s}$ in the formulae) extracted from the original cross. Following Jinks and Pooni (1976) we obtain these probabilities from the integrated density functions of

$$
\begin{gathered}
\int_{\overline{\mathrm{P}}_{1}}^{\infty} f(x) . d x ; \int_{-\infty}^{\overline{\mathrm{P}}_{2}} f(x) . d x ; \int_{\overline{\mathrm{F}}_{1}}^{\infty} f(x) . d x ; \int_{\overline{\mathrm{P}}_{l}}^{\infty} f(x) . d x \\
\text { and } \int_{-\infty}^{\overline{\mathrm{P}}_{s}} f(x) . d x
\end{gathered}
$$

respectively; and if the distribution of the recombinant hybrids is approximated with a standard normal distribution with mean " 0 " and variance " 1 ", then these integrals can be replaced by the one tail normal probability integral corresponding with the abscissa values of

$$
\begin{gathered}
\frac{[d]-\frac{1}{2}[h]}{\left(\frac{1}{2} D+\frac{1}{4} H+E\right)^{1 / 2}} ; \quad \frac{-[d]-\frac{1}{2}[h]}{\left(\frac{1}{2} D+\frac{1}{4} H+E\right)^{1 / 2}} ; \frac{\frac{1}{2}[h]}{\left(\frac{1}{2} D+\frac{1}{4} H+E\right)^{1 / 2}} ; \\
\frac{\sum d-\frac{1}{2}[h]}{\left(\frac{1}{2} D+\frac{1}{4} H+E\right)^{1 / 2}} \text { and } \frac{-\sum d-\frac{1}{2}[h]}{\left(\frac{1}{2} D+\frac{1}{4} H+E\right)^{1 / 2}}
\end{gathered}
$$

respectively for a situation where the estimates of the individuals parameters are available; or with the abscissa values of

$$
\frac{\overline{\mathrm{P}}_{1}-\overline{\mathrm{F}}_{2}}{\left(V \mathrm{~F}_{2}\right)^{1 / 2}} ; \quad \frac{\overline{\mathrm{P}}_{2}-\overline{\mathrm{F}}_{2}}{\left(V \mathrm{~F}_{2}\right)^{1 / 2}} ; \quad \frac{\overline{\mathrm{F}}_{1}-\overline{\mathrm{F}}_{2}}{\left(V \mathrm{~F}_{2}\right)^{1 / 2}} ; \quad \frac{\overline{\mathrm{P}}_{1}-\overline{\mathrm{F}}_{2}}{\left(V \mathrm{~F}_{2}\right)^{1 / 2}} ; \quad \frac{\overline{\mathrm{P}}_{s}-\overline{\mathrm{F}}_{2}}{\left(V \mathrm{~F}_{2}\right)^{1 / 2}}
$$

when the mean and variance of the $F_{2}$ generation are used as predictors.

These probabilities are valid for the phenotypic distribution of the recombinant hybrids but we can obtain predictions based solely on the repeatable genetic performance of these hybrids by excluding the nonrepeatable additive environmental variance, $E$, from the denominator before taking its square root, that is, by using $\left(\frac{1}{2} D+\frac{1}{4} H\right)^{1 / 2}$ instead of $\left(\frac{1}{2} D+\frac{1}{4} H+\right.$ $E)^{1 / 2}$ or $\left(V F_{2}-E\right)^{1 / 2}$ in place of $\left(V F_{2}\right)^{1 / 2}$.

\section{Complications}

(i) Genotype $\times$ environment interaction

In the presence of genotype $x$ environment interactions our estimates of the predictors $[d],[h], \frac{1}{2} D$ and $\frac{1}{4} H$ become $[d]+g_{d},[h]+g_{h}, \frac{1}{2} D+\frac{1}{2} G_{D}$ 
and $\frac{1}{4} H+\frac{1}{4} G_{H}$, respectively where $g_{d}, g_{h}, G_{D}$ and $G_{H}$ are the environmentally dependent components (Mather and Jinks, 1971, 1982). Estimates of the environmentally dependent components of the mean are readily obtained (Bucio Alanis, Perkins and Jinks, 1969) and estimates of the corresponding components of the variances are provided by the triple test cross (Perkins and Jinks, 1971), and approximations to them by the $F_{2}$ variance. These modified predictors, however, will correctly predict the properties of the recombinant $F_{1}$ hybrids only if there are no significant genotype $\times$ environment interactions between the environments in which the predictors are estimated and those in which the recombinant hybrids are assessed, that is, the two sets of environments can be regarded as having been drawn from the same population. This can be ascertained by growing the same controls, for example, the original parents and their $F_{1}$, in both sets of environments (see Jinks and Pooni, 1976).

\section{(ii) Linkage disequilibrium}

Provided that $K$ is large, linkage affects neither the actual nor the predicted mean of the second cycle recombinant hybrids in the absence of non-allelic interaction. It does, however, affect both the actual and predicted variances. Thus if $K$ is large the expected variance of the hybrids for many pairs of linked genes is

$$
\frac{1}{2} \sum d_{j}^{2}\left(\begin{array}{c}
+C \\
-R
\end{array}\right) \sum \frac{\left(1-2 p_{j k}\right)}{\left(1+2 p_{j k}\right)} d_{j} d_{k}+\frac{1}{4} \sum h_{j}^{2}+\frac{1}{2} \sum \frac{\left(1-2 p_{j k}\right)^{2}}{\left(1+2 p_{j k}\right)} h_{j} h_{k}
$$

using the definitions of gene effects of Mather and Jinks (1982).

On the same model the expectation of the predicted variance differ, with the source of the predictors (Mather and Jinks, 1982; Jinks and Pooni, 1981). For example, the genetic variance of the $F_{2}$ has the expectation

$$
\frac{1}{2} \sum d_{j}^{2}\left(\begin{array}{c}
+C \\
-R
\end{array}\right) \sum\left(1-2 p_{j k}\right) d_{j} d_{k}+\frac{1}{4} \sum h_{j}^{2}+\frac{1}{2} \sum\left(1-2 p_{j k}\right)^{2} h_{j} h_{k}
$$

while the estimate of $\frac{1}{2} D+\frac{1}{4} H$ from the $F_{2}$ triple test cross has the expectation

$$
\frac{1}{2} \sum d_{j}^{2}\left(\begin{array}{c}
+C \\
-R
\end{array}\right) \sum\left(1-2 p_{j k}\right) d_{j} d_{k}+\frac{1}{4} \sum h_{j}^{2}+\frac{1}{2} \sum\left(1-2 p_{j k}\right) h_{j} h_{k} .
$$

Linkage disequilibrium, that is an excess of coupling $(C)$ or repulsion $(R)$ linkages and of reinforcing ( $h_{j}$ and $h_{k}$ same sign) or opposing ( $h_{j}$ and $h_{k}$ opposite signs) dominance, rather than linkage per se determines the magnitude of the linkage bias of the actual and predicted variance of the second cycle hybrids. But in all circumstances this bias will be smaller in the actual than in the predicted variance. Our experience in predicting recombinant inbred lines (Jinks and Pooni, 1976, 1982; Pooni and Jinks, 1978; Pooni, Jinks and Cornish, 1977) shows that the differences in the biases are usually so small that they do not lead to significant departures between the actual and predicted variances. Furthermore, these differences can be reduced by using the genetic variance of a randomly mated $F_{2}$ as predictor (Jinks and Pooni, 1982). 
(iii) Epistasis

In the presence of epistasis the expectations of the mean and variance of the second cycle recombinant hybrids become

$$
m+\frac{2^{K-1}}{2^{K}-1}[h]+\frac{2^{K-2}}{2^{K}-1}(l)
$$

and

$$
\begin{aligned}
\frac{\left(2^{K-1}-1\right)}{\left(2^{K}-1\right)} D & +\frac{2^{K-1}\left(2^{K-1}-1\right)}{\left(2^{K}-1\right)^{2}} H-\frac{2^{K-1}}{\left(2^{K}-1\right)^{2}} \sum_{j<k}^{K} h_{j} h_{k}+\frac{2^{K-2}}{2^{K}-1}(I+2 J) \\
& +\frac{2^{K-2}}{\left(2^{K}-1\right)}\left(1-\frac{2^{K-2}}{\left(2^{K}-1\right)}\right) L+\text { cross products, }
\end{aligned}
$$

respectively (for definitions of $I, J$ and $L$, see Jinks, 1983). For many loci these statistics reduce to $m+\frac{1}{2}[h]+\frac{1}{4}[l]$ and $\frac{1}{2} D+\frac{1}{4} H+\frac{1}{4} I+\frac{1}{2} J+\frac{3}{16} L+$ cross products, which are the mean and variance of the $F_{2}$, respectively. Similarly, estimates of $m, \frac{1}{2}[h]$ and $\frac{1}{4}[l]$ obtained from the basic generations by maximum likelihood solutions and of $\frac{1}{2} D$ and $\frac{1}{4} H$ estimated from a triple test cross provide reasonably close approximation to the mean and variance of the second cycle, recombinant hybrids. However, as in the case of recombinant inbreds (Jinks and Pooni, 1976; Jinks, 1983) the major differences associated with the presence of non-allelic interactions are the shifts in the means of the five standard genotypes used in predictions (see section $3)$. The expectations of these genotypes now become:

$$
\begin{aligned}
& \overline{\mathbf{P}}_{1}=m+[d]+[i] \\
& \overline{\mathbf{P}}_{2}=m-[d]+[i] \\
& \overline{\mathbf{F}}_{1}=m+[h]+[l] \\
& \overline{\mathrm{P}}_{1}=m+\sum d+\sum i
\end{aligned}
$$

and

$$
\overline{\mathrm{P}}_{s}=m-\sum d+\sum i,
$$

respectively.

The consequences of these modifications can be readily seen. For example, if $[i], \sum i$ and $[l]$ take positive values the probabilities of the recombinant hybrids scoring $>\overline{\mathrm{P}}_{1},>\overline{\mathrm{P}}_{1}$ and $>\overline{\mathrm{F}}_{b}$, are reduced and the corresponding probabilities for $<\overline{\mathrm{P}}_{2},<\overline{\mathrm{P}}_{s}$ and $<\overline{\mathrm{F}}_{l}$ increased. The reverse of course is true when these components take negative values. Similarly, the probabilities associated with $>\overline{\mathbf{P}}_{1},>\overline{\mathrm{P}}_{1}$ and $>\overline{\mathrm{F}}_{1}$, are increased when $[h]$ and $[l]$ take opposing signs and reduced when $[l]$ reinforces $[h]$. To accommodate these changes, the definitions of the abscissa values associated with $>\overline{\mathbf{P}}_{1},<\overline{\mathrm{P}}_{2},>\overline{\mathrm{F}}_{1},>\overline{\mathrm{P}}_{1}$ and $<\overline{\mathrm{P}}_{s}$ are modified to become

$$
\begin{gathered}
\frac{[d]+[i]-\frac{1}{2}[h]-\frac{1}{4}[1]}{\left(\frac{1}{2} D^{\prime}+\frac{1}{4} H^{\prime}\right)^{1 / 2}}, \frac{-[d]+[i]-\frac{1}{2}[h]-\frac{1}{4}[l]}{\left(\frac{1}{2} D^{\prime}+\frac{1}{4} H^{\prime}\right)^{1 / 2}}, \frac{\frac{1}{2}[h]+\frac{3}{4}[l]}{\left(\frac{1}{2} D^{\prime}+\frac{1}{4} H^{\prime}\right)^{1 / 2^{\prime}}} \\
\frac{\sum d+\sum i-\frac{1}{2}[h]-\frac{1}{4}[l]}{\left(\frac{1}{2} D^{\prime}+\frac{1}{4} H^{\prime}\right)^{1 / 2}} \text { and } \frac{-\sum d+\sum i-\frac{1}{2}[h]-\frac{1}{4}[l]}{\left(\frac{1}{2} D^{\prime}+\frac{1}{4} H^{\prime}\right)^{1 / 2}}
\end{gathered}
$$

where $D^{\prime}$ and $H^{\prime}$ are the estimates of the additive and dominance genetic variances biased by the presence of non-allelic interactions. 


\section{(iv) Non-normality}

Our predictions assume that the genotypic distribution of the recombinant hybrids is approximately normal. This will be true, however, only where all the known sources of non-normality e.g., dominance, epistasis, linkage disequilibrium and genotype $\times$ environmental interactions are either non significant or the non-normalities they produce balance out (Jinks and Pooni, 1976; Pooni, Jinks and Cornish, 1977; Jinks, 1983). In general we therefore expect to find some non-normality in the distribution of hybrids and this may affect the accuracy of the predictions, especially when the probabilities take large values. Previous experience in predicting the properties of recombinant inbred lines suggests that the levels of epistasis, linkage disequilibrium and genotype $\times$ environment interactions encountered in practice are unlikely to cause difficulties. In predicting the properties of recombinant hybrids, however, directional dominance is potentially a more serious source of non-normality but even this is not expected to cause difficulties unless the number of loci involved is small and the dominance ratio is high.

\section{EXPERIMENTS AND RESULTS}

\section{(i) Predicted properties}

To make and test the predictions we shall use material derived from the cross between varieties 1 and 5 of Nicotiana rustica. This cross, initiated by Mather and Vines in 1952, provides data on the parental varieties and their $F_{1}$ and $F_{2}$ generations for most of the intervening years. To these sources of data we must add the extreme families present amongst a random sample of 82 inbred lines extracted from the $F_{2}$ of this cross by single seed descent (for further information see Perkins and Jinks, 1973; Jinks and Pooni, 1976). As the scores of these inbreds are available together with $V_{1}, V_{5}, F_{1}$ and $F_{2}$ for $1973,1978-81$ and 1983 only we shall use the data from these seasons for making predictions. References to the sources of these data and the structure of each experiment is given in table 1.

The number and detail of the characters scored in Nicotiana rustica experiments vary with the objectives and their potential usefulness. All are scored however for flowering time and final height. The overall mean

\section{TABLE 1}

The sources, structure and dimensions of the data used to estimate the statistics for making predictions

\begin{tabular}{|c|c|c|c|c|c|c|c|}
\hline \multirow[b]{2}{*}{ Year } & \multirow[b]{2}{*}{ Source } & \multicolumn{4}{|c|}{ Family size } & \multirow[b]{2}{*}{$P_{\text {largest }}$} & \multirow[b]{2}{*}{$P_{\text {smallest }}$} \\
\hline & & $v_{s}$ & $\mathrm{~V}_{\mathfrak{l}}$ & $F_{1}$ & $\mathrm{~F}_{2}$ & & \\
\hline 1973 & Pooni, Jinks and & & & & & & \\
\hline & Jayasekara (1978) & 140 & 120 & 140 & 240 & 10 & 10 \\
\hline 1978 & Authors & 40 & 40 & 40 & 150 & 10 & 10 \\
\hline 1979 & Al-Banna (1983) & 25 & 25 & 50 & 820 & 10 & 10 \\
\hline 1980 & Authors & 30 & 30 & 60 & 60 & 10 & 10 \\
\hline 1981 & Authors & 30 & 30 & 60 & 120 & 10 & 10 \\
\hline 1983 & Authors & 20 & 20 & 40 & 25 & 10 & 10 \\
\hline
\end{tabular}


performances of $V_{1}, V_{5}, F_{1}, F_{2}, P_{\text {largest }} P_{\text {smallest }}$ families and the mean genetic $\left(\mathrm{V}_{1} \mathrm{~F}_{2}\right)$ and environmental $(E)$ components of variance averaged over the 6 environments (table 1) are given in table 2. Scores for flowering time represent the number of days taken to flower from an arbitrary date and the final height measurements are presented in $\mathrm{cm}$.

TABLE 2

Averaged estimates of the first and second degree statistics required for making predictions about the mean performance of second cycle hybrids and their predicted properties in respect of flowering time and final height

\begin{tabular}{|c|c|c|}
\hline Source & Flowering time & Final height \\
\hline \multicolumn{3}{|c|}{ (a) Family means } \\
\hline$P_{\text {largest }}$ & $39 \cdot 11$ & $151 \cdot 59$ \\
\hline$P_{\text {smallest }}$ & 14.43 & $87 \cdot 73$ \\
\hline$V_{5}$ & $23 \cdot 20$ & $126 \cdot 11$ \\
\hline$v_{1}$ & $20 \cdot 22$ & $112 \cdot 46$ \\
\hline $\mathrm{F}_{1}$ & $22 \cdot 02$ & $138 \cdot 86$ \\
\hline $\mathrm{F}_{2}$ & $23 \cdot 13$ & $129 \cdot 96$ \\
\hline \multicolumn{3}{|c|}{ (b) Components of variances } \\
\hline Genetic $\left(\mathrm{V}_{1} \mathrm{~F}_{2}\right)$ & $19 \cdot 79$ & $99 \cdot 34$ \\
\hline Environmental (E) & 14.97 & $108 \cdot 58$ \\
\hline \multicolumn{3}{|c|}{ (c) Predicted proportions } \\
\hline$>\overline{\mathbf{P}}_{1}$ & 0.0002 & 0.015 \\
\hline$<\overline{\mathbf{P}}_{s}^{\prime}$ & 0.0253 & 0.00001 \\
\hline$>\overline{\mathrm{V}}_{5}^{s}$ & 0.494 & 0.650 \\
\hline$<\overline{\mathrm{V}}_{1}^{3}$ & $0 \cdot 256$ & 0.040 \\
\hline$>\overline{\mathbf{F}}_{1}^{\prime}$ & 0.599 & 0.187 \\
\hline$<\overline{\mathrm{F}}_{1}$ & 0.401 & 0.813 \\
\hline
\end{tabular}

Predictions were made from these estimates using the formulae described in section 3 based upon the square root of the genetic variance, as opposed to the phenotypic variance, as denominator. The probabilities thus obtained for the two most important categories $>\overline{\mathrm{P}}_{1}$ and $<\overline{\mathrm{P}}_{s}$ are tabulated in the lower half of table 2 together with the predicted proportions of recombinant hybrids that should score $>\overline{\mathrm{V}}_{5},<\overline{\mathrm{V}}_{1},>\overline{\mathrm{F}}_{1}$ and $<\overline{\mathrm{F}}_{1}$.

\section{(ii) Observed proportions}

We shall test the reliability of the prediction process by comparing the observed and predicted proportions of second cycle recombinant hybrids that fall within the specified classes. The observed proportions will be taken from a $20 \times 20$ diallel produced from a random sample of $20 \mathrm{~F}_{9}$ recombinant inbred lines each of which was extracted from a separate $F_{2}$ plant of the $V_{1} \times V_{5}$ cross by pedigree inbreeding. The diallel crosses and selfs were produced during 1954 and the experiment conducted during 1955 by $\mathrm{Dr}$ E. L. Breese. Experimental details and results from this diallel have already been published in one form or another by Jinks, Perkins and Breese (1969), Virk and Jinks (1977) and Pooni, Jinks and Singh (1984).

The scores of the 20 inbred parents and 190 crosses after averaging over reciprocals can be used directly to obtain two sets of observed proportions. For example, for flowering time we can select the earliest and latest flowering 
inbred out of the 20 inbred lines and then count the number of hybrids which either flower earlier than the earliest inbred or flower later than the latest flowering inbred. The $P_{\text {largest }}$ and $P_{\text {smallest }}$ were therefore different from those used to make the predictions, as the latter were not, of course, available in 1955. Normally, of course, they would be the same families. In comparing the predicted and observed proportions we shall, therefore, be introducing an additional source of error.

The observed numbers of hybrids (out of 190) which either score $\geqq \overline{\mathrm{P}}_{l}$ or $\leqq \overline{\mathrm{P}}_{s}$ or fall within their range are tabulated both for flowering time and final height in table 3 , along with the predicted numbers for each of the three categories rounded off as whole numbers.

Since the parental varieties and their $F_{1}$ generation were not part of the $20 \times 20$ diallel, the remaining comparisons between observed and expected proportions cannot be made. However, observed proportions obtained by extrapolating the scores of $V_{5}, V_{1}$ and $F_{1}$ families, assuming that they show linear responses to an environmental index defined by deviations in the overall means of the $\mathrm{F}_{\infty}$ inbred lines, compared favourably with the predictions given in table 2 .

\section{Conclusions}

In this paper we have shown how to predict the properties of second cycle $F_{1}$ hybrids which can be produced by intercrossing a random sample of inbred lines extracted from the $\mathrm{F}_{2}$ of a cross by single seed descent or pedigree inbreeding. We have shown (sections 3 and 4 ) that these predictions can be made by using genetical components that are most readily and reliably estimated in the early stages of a breeding programme. In fact data from the $F_{2}$ generation of the original cross is all that is required to predict the expected mean and variance of these hybrids. With this information we can determine the probabilities of obtaining hybrids whose scores fall into any phenotypic class we care to specify. In the early generations of a cross we can now, therefore, simultaneously predict the distribution of the recombinant inbred lines and of the second cycle hybrids that can be derived from them. Hence we can assess the breeding value of a cross whether the intended end products of the breeding programme are superior inbred lines or superior hybrids. We can also predict which would be the better end product in terms of performance and whether a second cycle of extraction of recombinant inbred lines is likely to produce a significant improvement on the first.

At first sight linkage and genotype $\times$ environment interaction may appear to complicate the procedures for making reliable predictions (see section 3) but in general they produce only relatively minor distortions whose presence can be detected. While, therefore, they have some effect on the quantitative accuracy of the predictions no adjustments will usually be required in the prediction procedures to accommodate their effects.

In contrast, non-allelic interactions can produce distortion of a magnitude which must be allowed for in making predictions. But, as we have already shown in predicting the properties of recombinant inbred lines (Jinks and Pooni, 1976; Pooni, Jinks and Cornish, 1977), the major source of these biases arises from the non-allelic interaction components of the means $[i]$ and $[l]$ and these can be estimated and allowed for when making 
the predictions. These components are automatically accommodated in the predictors when these are the mean and variance of the $F_{2}$ but they must be explicitly allowed for when the predictors are combinations of genetical components estimated from the early generations by weighted least squares.

TABLE 3

Predicted frequencies of the recombinant hybrids falling into the phenotypic classes specified by the scores of the largest and smallest scoring inbred lines and the correspondingly observed frequencies amongst 190 hybrids extracted from a $20 \times 20$ diallel

\begin{tabular}{ccccc}
\hline & \multicolumn{2}{c}{ Flowering time } & \multicolumn{2}{c}{ Final height } \\
\cline { 2 - 5 } Category & Predicted & Observed & Predicted & Observed \\
\hline$>\overline{\mathrm{P}}_{l}$ & 0 & 0 & 3 & 1 \\
$<\overline{\mathrm{P}}_{s}$ & 5 & 9 & 0 & 0 \\
Remainder & 185 & 181 & 187 & 189 \\
\hline
\end{tabular}

The main conclusion to emerge from the analyses (section 4 ) and results (table 3 ) is that the prediction procedures are reasonably robust. Thus in spite of modest levels of non-allelic interaction, linkage disequilibrium and genotype $\times$ environment interaction (Pooni, Jinks and Jayasekara, 1978; Jinks, Perkins and Pooni, 1973; Perkins and Jinks, 1970) and the use of different pairs of extreme recombinant inbred genotypes for making the predictions and for assessing them, the predictions are consistent with the results obtained (table 3 ). The probabilities used in the predictions, however, are small enough to be determined by the extremes of the expected and observed distributions of the hybrids and are, therefore, less influenced by the distortions in the body and shoulders of the distributions.

The present study also shows that, even where the best recombinant inbred lines are far superior to the orginal heterotic $F_{1}$, it is still relatively easy to produce second cycle hybrids that perform better than the best inbred provided that they lie in the direction of the dominance, that is, greater final height, earlier flowering (tables 2 and 3). Equally, however, the standard procedures for predicting the distribution of recombinant inbred lines show that we can expect to extend the range of performances by a second cycle of inbreeding. Thus we expect a proportion of 0.0001 $>\overline{\mathbf{P}}_{l}$ and $0.017<\overline{\mathrm{P}}_{s}$ for flowering time and $0.05>\overline{\mathrm{P}}_{l}$ and $0.017<\overline{\mathrm{P}}_{s}$ for final height among a random sample of second cycle recombinant inbred lines. These proportions are close to those for superior second cycle hybrids (table 2). It is possible, therefore, that these superior second cycle hybrids are very similar in genotype to the superior second cycle inbreds, that is, they may be homozygous for the same alleles at most loci and heterozygous at only a very few.

Acknowledgements. J. F. F. de Toledo is on leave from Centro Nacional de Pesquisa de Soja-Embrapa, Caixa Postal 1061, 86100-Londrina-Pr, Brazil.

\section{REFERENCES}

AL-BANNA, M. K. S. 1983. Selection for environmental sensitivity and competitive ability in Nicotiana rustica. Ph.D. Thesis, University of Birmingham. 
BUCIO ALANiS, L., PERKINS, J. M. AND JiNKS, J. L. 1969. Environmental and genotype $x$ environmental components of variability. V. Segregating generations. Heredity, 24, 115-1 27.

JINKS, J. L. 1983. Biometrical genetics of heterosis. In Frankel, R. (ed.) Heterosis, SpringerVerlag, Berlin, pp. 1-46.

JINKS, J. L., PERKINS, J. M. AND BREESE, E. L. 1969. A general method of detecting additive, dominance and epistatic variation for metrical traits. II. Application to inbred lines. Heredity, 24, 45-57.

JINKS, J. L., PERKINS, J. M. AND POONI, H. S. 1973. The incidence of epistasis in normal and extreme environments. Heredity, 31, 263-269.

JINKS, J. L. AND POONI, H. S. 1976. Predicting the properties of recombinant inbred lines derived by single seed descent. Heredity, 36, 253-266.

JINKS, J. L. AND POONI, H. S. 1980. Comparing predictions of mean performance and environmental sensitivity of recombinant inbred lines based upon $\mathrm{F}_{3}$ and triple test cross families. Heredity, 45, 305-312.

JINKS, J. L. AND POONI, H. S. 1981. Properties of pure breeding lines produced by dihaploidy, single seed descent and pedigree inbreeding. Heredity, 46, 391-395.

JINKS, J. L. AND POONI, H. S. 1982. Predicting the properties of pure breeding lines extractable from a cross in the presence of linkage. Heredity, 49, 265-270.

KEARSEY, M. J. AND JINKS, J. L. 1968. A general method of detecting additive, dominance and epistatic variation for metrical traits. I. Theory. Heredity, 23, 403-409.

MATHER, K. AND JINKS, J. L. 1971. Biometrical Genetics. 2nd Edition. Chapman and Hall, London.

MATHER, K. AND JiNkS, J. L. 1982. Biometrical Genetics. 3rd Edition. Chapman and Hall, London.

MATHER, K. AND VINES, A. 1952. The inheritance of height and flowering time in a cross of Nicotiana rustica. In Reeve, E. C. R. and Waddington, C. H. (eds.) Quantitative Inheritance, HMSO, London, pp. 49-80.

PERKINS, J. M. AND JINKS, J. L. 1970. Detection and estimation of genotype-environmental, linkage and epistatic components for a metrical trait. Heredity, 25 157-177.

PERKINS, J. M. AND JINKS, J. L. 1971. Analysis of genotype $\times$ environment interaction in triple test cross data. Heredity, 26, 203-209.

PERKINS, J. M. AND JINKS, J. L. 1973. The assessment and specificity of environmental and genotype-environmental components of variability. Heredity, 30, 111-126.

POONI, H. S. AND JINKS, J. L. 1976. The efficiency and optimal size of triple test cross designs for detecting epistatic variation. Heredity, 36, 215-227.

POONI, H. S. AND JINKS, J. L. 1978. Predicting the properties of recombinant inbred lines derived by single seed descent for two or more characters simultaneously. Heredity, 40, 349-361.

POONI, H. S. AND JINKS, J. L. 1981. Sources of predictions and their reliability in predicting the properties of recombinant inbred lines which can be obtained from a cross by single seed descent. Proceedings of the Fourth International Barley Genetics Symposium, Edinburgh, Scotland, pp. 73-78.

POONI, H. S., JINKS, J. L. AND CORNISH, M. A. 1977. The causes and consequences of non-normality in predicting the properties of recombinant inbred lines. Heredity, 38, 329-338.

POONI, H. S., JINKS, J. L. AND JAYASEKARA, N. E. M. 1978. An investigation of gene action and genotype $X$ environment interaction in two crosses of Nicotiana rustica by triple test cross and inbred line analysis. Heredity, 41, 83-92

POONI, H. S. JINKS, J. L. AND SINGH, R. K. 1984. Methods of analysis and the estimation of the genetic parameters from a diallel set of crosses. Heredity, 52, 243-253

VIRK, D. S. AND JINKS, J. L. 1977. The consequences of using inadequate testers in the simplified triple test cross. Heredity, 38, 237-251. 